\title{
Development of the Wind Tunnel CFD Model for Clarifying Rotor Blade Airfoil Unsteady Aerodynamic Researches
}

\author{
Oleg E. Kirillov ${ }^{1}$, Ruslan M. Mirgazov ${ }^{1}$, Yuri M. Ignatkin ${ }^{2}$, \\ Sergey G. Konstantinov ${ }^{2}$, Pavel V. Makeev ${ }^{2}$, Mikhail Yu. Buntov ${ }^{2}$ \\ ${ }^{1}$ Central Aerohydrodynamic Institute (TsAGI), 140180, Moscow Oblast, \\ Zhukovsky street, 1, Zhukovsky, Russia \\ ${ }^{2}$ Moscow Aviation Institute (National Research University), Volokolamskoe Highway, 4, \\ 125993, Moscow, Russia
}

\begin{abstract}
A computational model of a wind tunnel (WT) with a special experimental rig has been developed directly for studying unsteady aerodynamic characteristics of a wing section with a helicopter blade profile. The model was constructed using CFD (Computational Fluid Dynamics) methods based on the URANS approach (Unsteady Reynolds Averaged Navier Strokes Equations). Therefore, the aerodynamic characteristics of the airfoil can be determined, taking into account influence of the WT walls such as walls perforation and various configurations of the experimental rig. Simulation of the flow around the wing section with the SC1095 airfoil in steady and unsteady settings is performed. The flow features in the working section of the WT and the experimental rig are analyzed. For a particular case, the calculation method was validated in a 2D formulation on the basis of available experimental data. The developed model can be used to refine the methods of processing experimental data, taking into account the individual characteristics of the WT and the experimental rig configuration.
\end{abstract}

DOI: $10.18421 /$ TEM102-10

https://doi.org/10.18421/TEM102-10

Corresponding author: Mikhail Yu. Buntov, Moscow Aviation Institute (National Research University), Moscow, Russia.

Email: mikhail.yu.buntov@mail.ru

Received: 19 November 2020.

Revised: 26 April 2021.

Accepted: 30 April 2021.

Published: 27 May 2021.

(cc) BY-NC-ND (C) 2021 Oleg E. Kirillov et al; published by UIKTEN. This work is licensed under the Creative Commons Attribution-NonCommercial-NoDerivs 4.0 License.

The article is published with Open Access at www.temjournal.com
Keywords - aerodynamic characteristics; CFD method; helicopter blade airfoil; unsteady flow; wind tunnel.

\section{Introduction}

The main rotor blades of the helicopter operate in a variable velocity field, which causes periodic flapping motions of the blades relative to horizontal hinges or alternative elastic elements. The phenomenon of unsteady flow around the blade airfoil is observed [1]. Unsteady aerodynamic characteristics associated with a cyclic change in the airfoil angle of attack and incoming flow velocity differ significantly from the steady aerodynamic characteristics obtained at constant values of the angles of attack and flow velocity [2]. It is important to take into account unsteady flow when calculating the aerodynamic characteristics of the main rotor, especially at high speeds of horizontal flight. To study the unsteady flow around the airfoil, as a rule, it is necessary to conduct complex experimental studies in wind tunnel (WT). In this case, the wing section with the studied airfoil is installed in the WT in a specialized rig, which ensures its cyclic oscillation according to a given law [3]. As a result of processing experimental data, unsteady airfoil aerodynamic characteristics are obtained, which are then used in various methods for calculating the aerodynamic characteristics of the helicopter's main rotor. Experimental work on the study of unsteady flow around a wing with a helicopter airfoil is widely carried out at Central Aerohydrodynamic Institute named after Professor N.E. Zhukovsky [4], [5].

In recent years, the development of CFD methods and computer technology makes it possible to supplement experimental studies of various problems of aerodynamics with numerical modeling using math models of WT of various configurations. This direction is used both in aviation, for example, in the 
study of a tiltrotor aerodynamics [6], [7], and in industrial aerodynamics, for example, in the automobile industry [8], [9] and in the creation of wind generators [10], [11]. CFD methods are successfully used both for modeling the flow in subsonic and studying supersonic aerodynamics [12]. Modeling of the airfoil's aerodynamic characteristics with special attention to the influence of the working section's walls of WT was also previously performed in TSAGI [13]. In addition, CFD methods are also used in the design of WT [14].

The paper describes the development of the math model of working section of the WT with a specialized rig installed inside to study the unsteady flow around the wing section with a helicopter airfoil. The developed model makes it possible to study the flow features in the WT and rig path, as well as to conduct numerical simulation of the experiment. This approach makes it possible to refine the method of processing experimental results and

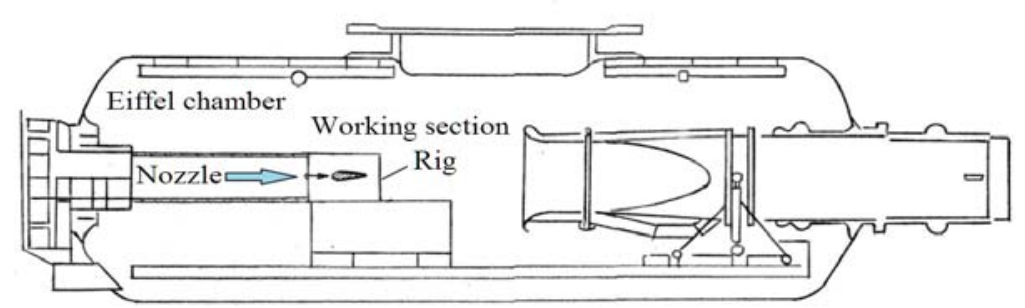

a) Scheme of the WT take into account the individual features of the experimental rig in its various configurations [15].

\section{Methodology}

\subsection{Object of Study}

The closed-type WT (Figure 1.a.) is designed for experimental study of the aerodynamic characteristics of aircraft models and airfoils in a wide range of Mach numbers. The experimental rig installed in the working section of the WT (Figure 1.b.) has a rectangular cross-section with dimensions of $0,5 \times 0,52 \mathrm{~m}$ and provides cyclical change of the wing section pitch angle during the experiment according to a predetermined law. The model of the wing section can rotate relative to the axis located at a distance of a quarter of chord from the airfoil's leading edge.

Figure 1. Scheme of the WT and photo of the experimental rig with the wing section installed

In the case of studies of the oscillating wing section, the pitch angle changes according to the following law:

$$
\alpha(t)=\alpha_{a v}+A_{V} \sin \left(\omega t+\varphi_{i}\right)
$$

where $\alpha_{a v}$ is average pitch angle, $\psi(t)=\omega t$ is angle of the wing oscillation drive shaft rotation, $\omega=2 \pi f[1 / \mathrm{c}]$ is circular frequency, $f[\mathrm{~Hz}]$ is frequency of rotation of the oscillation drive shaft, respectively (angular frequency and wing oscillation frequency), $A_{V}$ and $\varphi_{\mathrm{i}}$ is amplitude and initial phase angle of the wing oscillation.
The size of the working section of the WT is limited.

With a significant overlap of the working section area, the effect of "locking" is observed. The "locking" effect is illustrated in Figure 2 - it demonstrates the distribution of static pressure in the cross section of the experimental rig working section. These results are obtained on the basis of the math model of the WT and the rig in the configuration "without walls perforation". It can be seen that at large values of wing section pitch angle the area of increased pressure covers the entire area of the WT working section "locking it", making it difficult to conduct the experiment. 


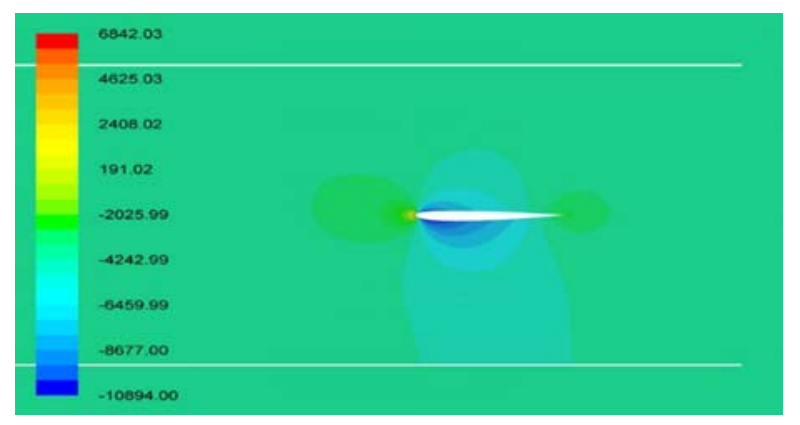

a) pitch angle $\alpha=0^{\circ}$

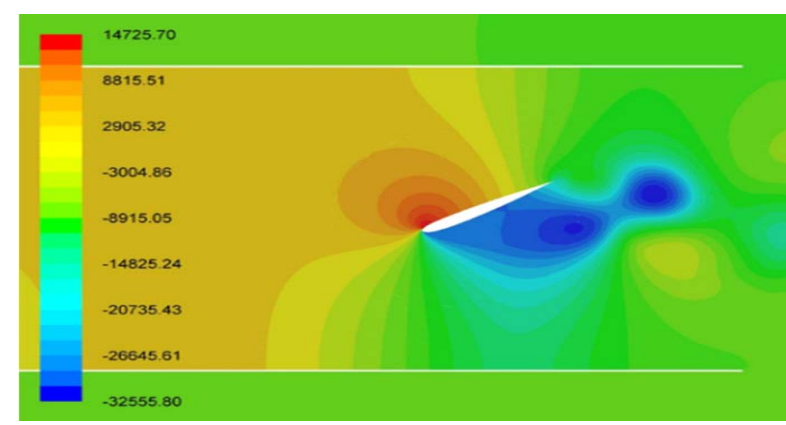

b) pitch angle $\alpha=28^{\circ}$

Figure 2. Distribution of static pressure in the longitudinal section of the of the experimental rig in the WT (without walls perforation)

To avoid the "locking" effect, 7\% perforation of the walls of the WT nozzle is used. Various variants of the experimental rig configuration are considered: with solid walls of the rig; with the upper perforated cover; without the upper cover. The math model of the WT and the experimental rig described below can contribute to a comprehensive study of these effects.

\subsection{The Mathematical Model}

Numerical simulation was performed in the ANSYS FLUENT software package using the unsteady RANS method and the k- $\omega$ SST turbulence model. To make a computational mesh in ANSYS ICEM, a 3D model of the WT was previously created. The model consists of five parts: the adaptable nozzle, the perforated nozzle, the working section (with experimental rig), the telescopic collector and the Eiffel chamber (Figure 3). To optimize the number of cells and reduce the required computational resources, a hybrid mesh consisting of structured and unstructured meshes is used. Thus the computational volume is divided into several parts between which sliding interfaces are used.

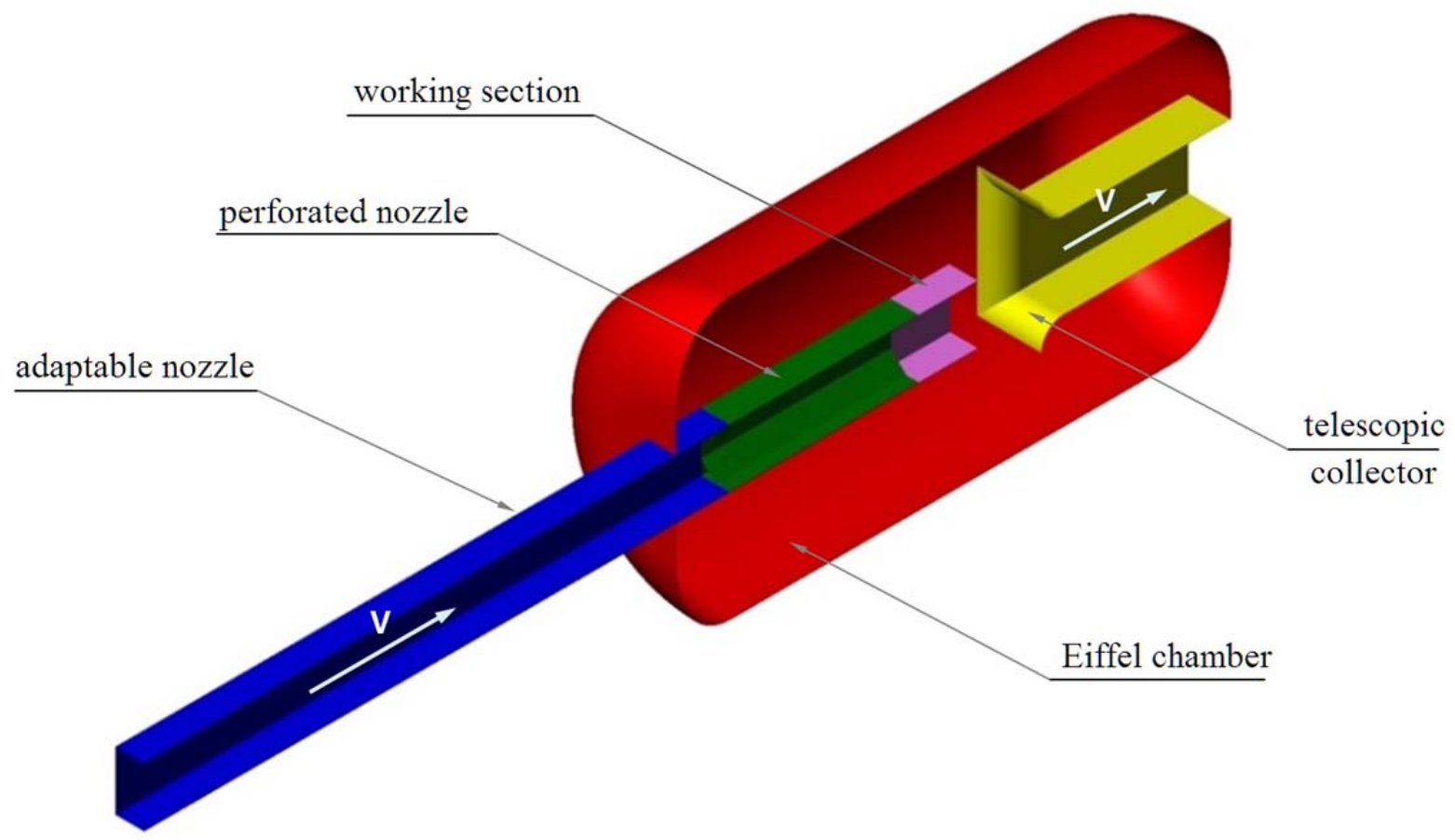

Figure 3. 3D-model of the WT

The first area (Figure 4) consists of the Eiffel chamber and rectangular inlet nozzle.
The area is a block-structured mesh with a total of $3 \times 10^{6}$ cells. 


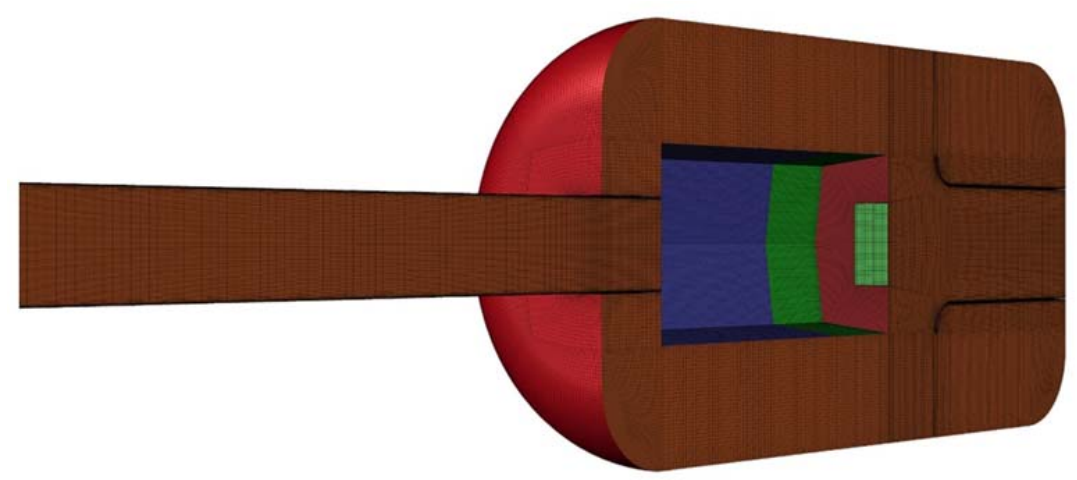

Figure 4. Surface mesh of the first area (inlet nozzle and Eiffel chamber)

The second area (Figure 5) simulates a perforated nozzle and is made in the form of an unstructured mesh with the number of cells $11.2 \times 10^{6}$. The third area (Figure 6) was modeled as the experimental rig with a top perforated cover, the total number of cells in this area is $1.2 \times 10^{6}$.

The fourth area (Figure 7) was a cylinder with a wing section located inside with the SC 1095 airfoil.

The geometric shape of this area is chosen taking into account the modeling of various pitch angles and periodic airfoil fluctuations. The number of cells in this area is $2.3 \times 10^{6}$. Area No. 2 and No. 3 were placed in area No. 1 their merging was through the use of the sliding interface. Area No. 4 was placed in area No. 3 by the same way.

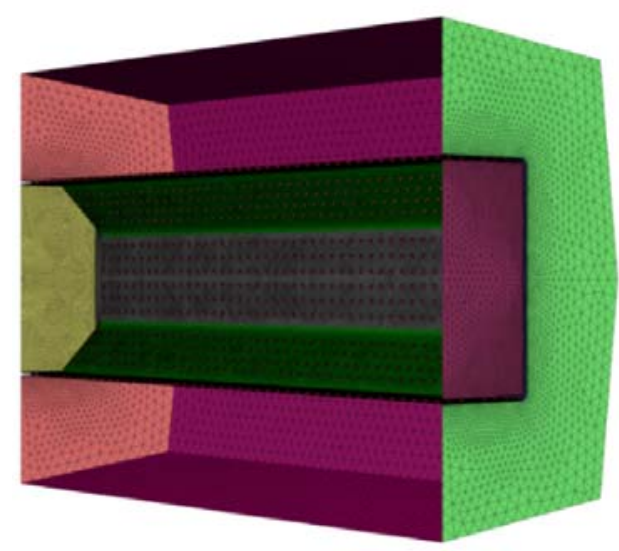

a) General view of the calculation area

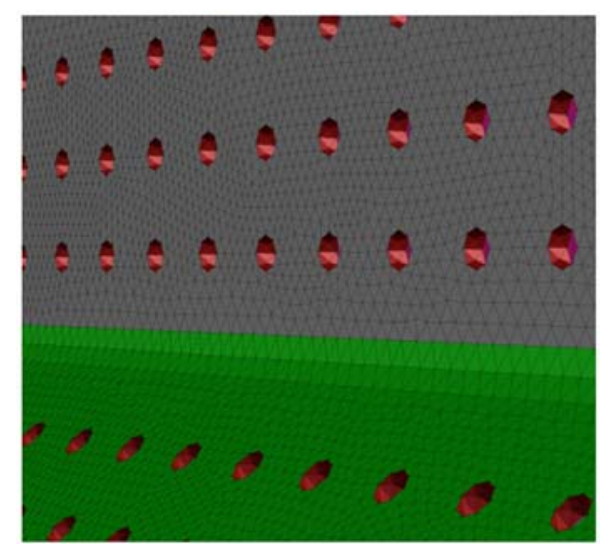

b) Surface mesh in the perforation zone

Figure 5. Second area (perforated nozzle)

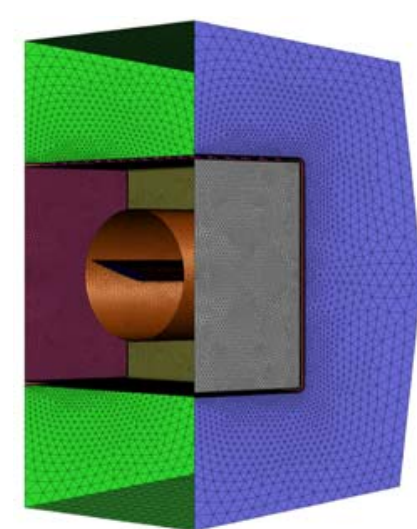

Figure 6. Surface mesh of the third area (experimental rig)

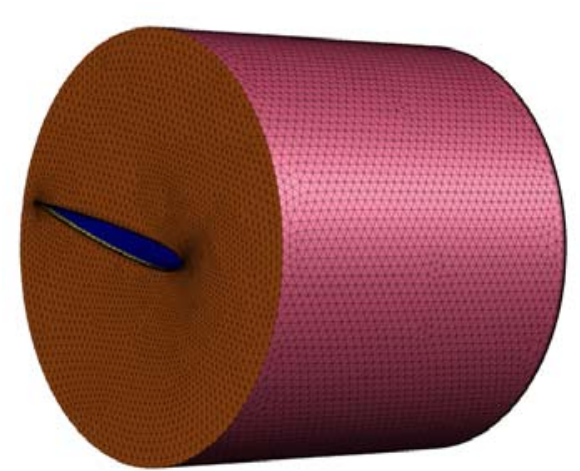

Figure 7. Surface mesh of the fourth area (wing section)

Figure 8 shows the total computational mesh of the WT and experimental rig.

Described method of mesh creating not only reduces the number of cells, but also allows creating new configurations of the WT, the experimental rig, and the model under study by replacing individual mesh parts. This approach allows rebuilding only certain areas of the computational model, which reduces the preparation time of the computational experiment. 


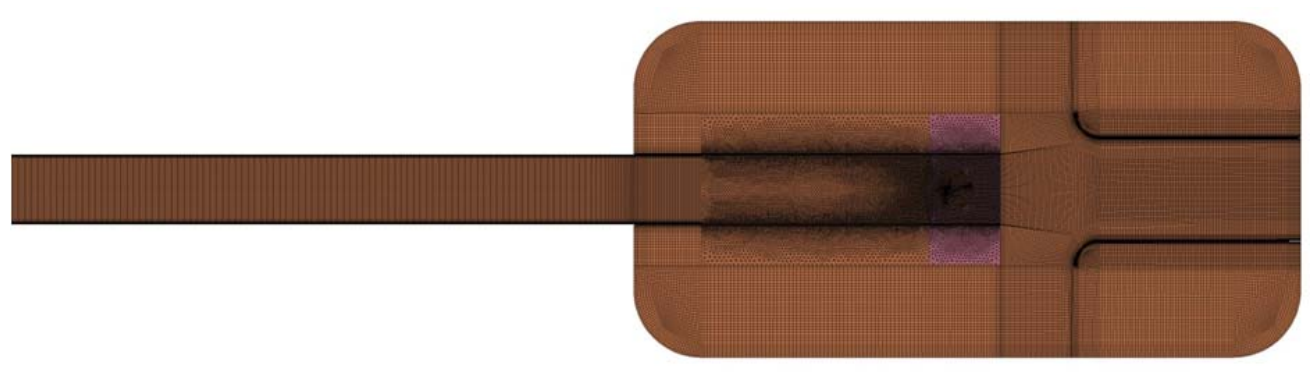

Figure 8. Total computational mesh

\subsection{Validation of the applied CFD method}

The SC1095 airfoil under study was pre-calculated in a $2 \mathrm{D}$ formulation in order to verify the CFD method used for calculating aerodynamic characteristics [16]. For this purpose, a 2D computational domain with a block-structured mesh topology was created in the ANSYS ICEM software (see Figure 9). The height of the cell adjoining to the surface was selected so that the first grid node from the wall was located in the viscous sublayer of the velocity profile $\left(\mathrm{Y}^{+} \leq 1\right)[17]$. The calculated area with the SC1095 airfoil having a chord $\mathrm{c}=0.4064 \mathrm{~m}$ in the center, and with external borders located at a distance of 16 chords from the airfoil, is shown in Figure 10. Total number of cells in the mesh is about 390000. In the process of calculation there has been used the k- $\omega$ SST turbulence model [18]. The initial turbulence parameters were selected based on the conditions of low intensity of the turbulent flow. The value of the relative turbulent viscosity was assumed to be $\mu_{t} / \mu=1$, and the turbulence intensity $I=1 \%$. Calculations of the aerodynamic characteristics of the SC1095 airfoil for angles of attack $-8^{\circ} \leq \alpha \leq 10^{\circ}$ were performed in a steady formulation, and for angles of attack $\alpha \geq 10^{\circ}-$ in unsteady one.
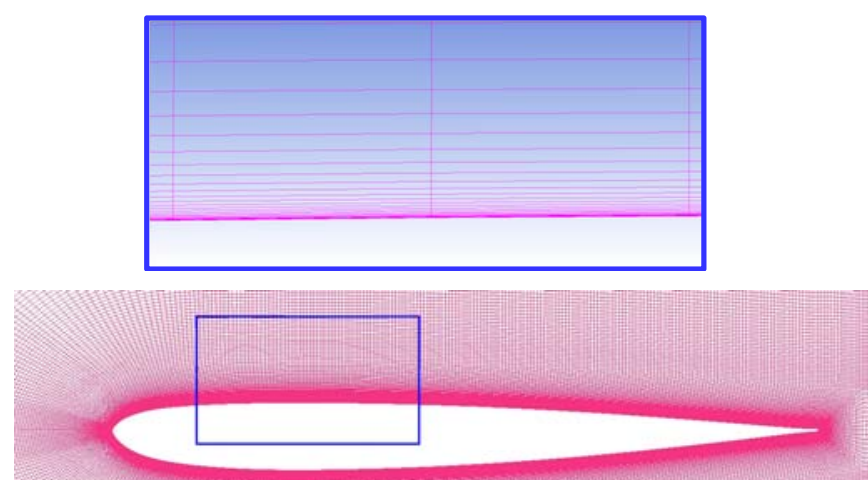

Figure 9. Structure of the computational mesh in the SC1095 airfoil area

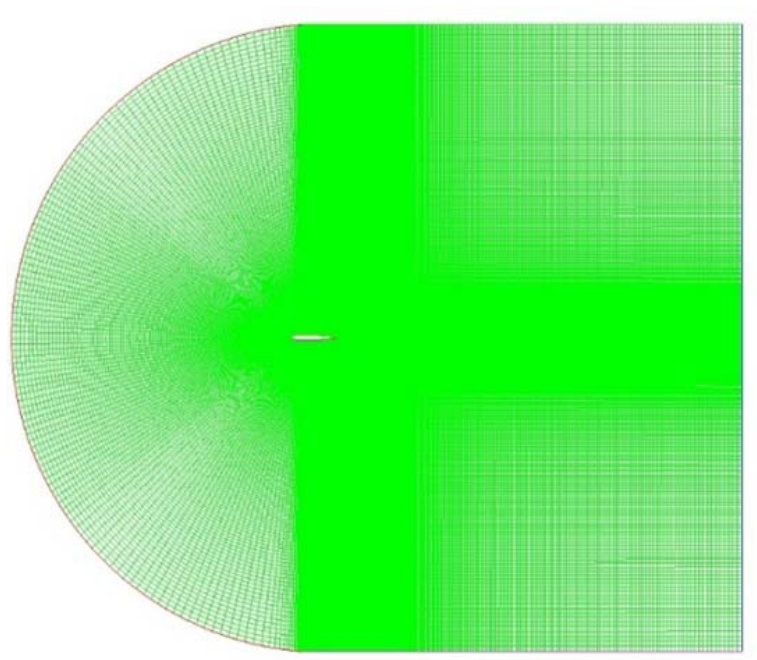

Figure 10. Computational area for 2D modeling of the flow around the SC1095 airfoil

Figure 11 shows the calculated and experimental dependences of the drag coefficient of the SC1095 airfoil obtained with the zero value of the lift coefficient $C_{L}=0$ in the range of Mach numbers $\mathrm{M}=$ $0.1 \ldots 1.1$.

The graph shows a significant increase in the values of the calculated drag coefficient at Mach number $M \geq 0.8$. The results of the calculations are in satisfactory agreement with experimental data [19]. Figure 12 and Figure 13 show the calculated and experimental values of the drag and lift coefficients $C_{\mathrm{D}}$ and $C_{\mathrm{L}}$ of SC1095 airfoil for the Mach number M $=0.4$ depending on the angle of attack $\left(C_{\mathrm{D}}\right.$ and $C_{\mathrm{L}}$ are in the flow coordinate system, where the OX axis coincides with the flow axis).

The results of calculations for the dependencies $C_{\mathrm{D}}$ $=f(\alpha)$ and $C_{\mathrm{L}}=f(\alpha)$ are close to the experimental data in the range of pitch angles $-5^{\circ} \leq \alpha \leq 12^{\circ}$ [20]. At angles of attack $\alpha>12^{\circ}$, the calculated dependences $C_{\mathrm{D}}=f(\alpha)$ and $C_{\mathrm{L}}=f(\alpha)$ differ to some degree from the experimental, but the type of change in the pitch angle is preserved. 


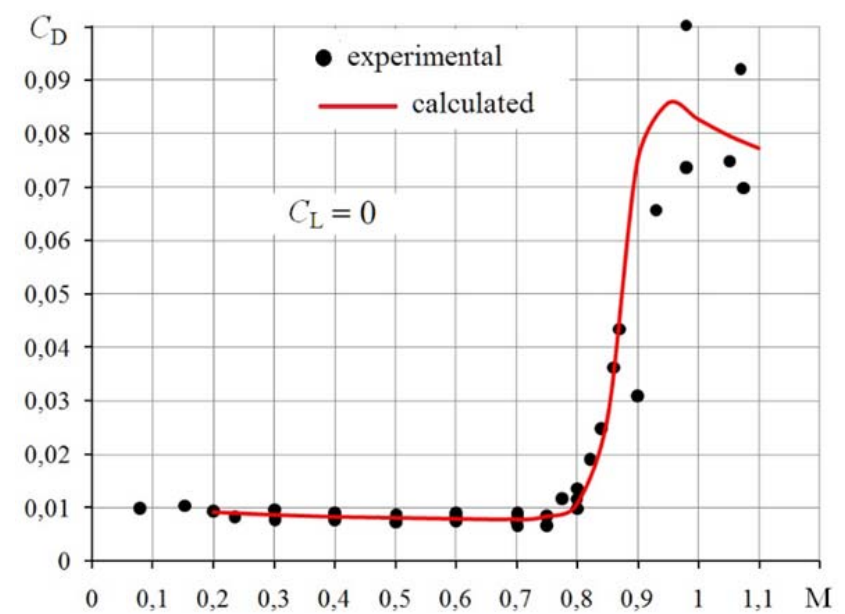

Figure 11. Calculated and experimental dependences of the drag coefficient of the SC1095 airfoil on the Mach number at $C_{L}=0$

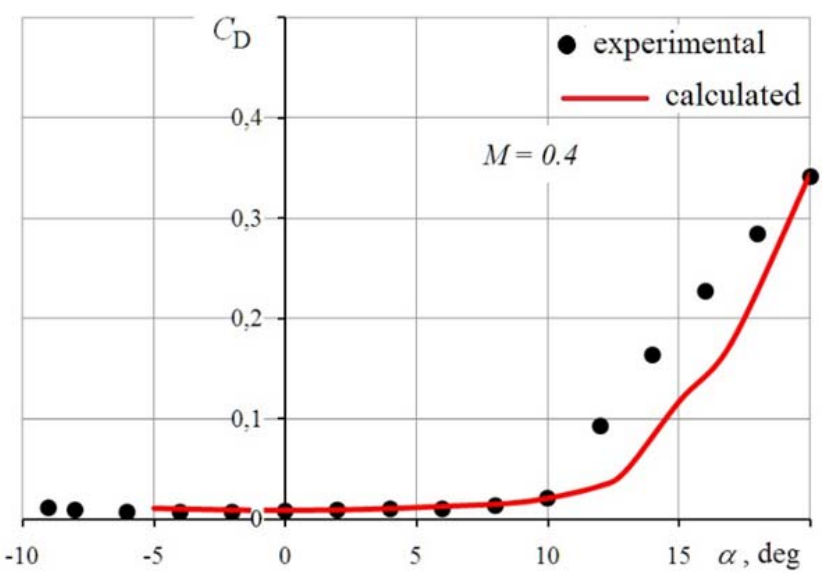

Figure 12. Dependence of the drag coefficient of the SC1095 airfoil on the pitch angle

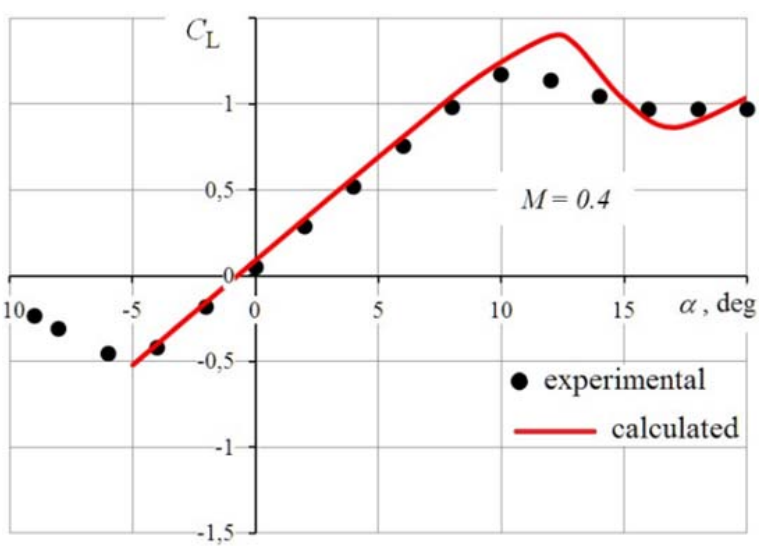

Figure 13. Dependence of the lift coefficient of the SC1095 airfoil on the pitch angle

\section{A) Results and Discussion}

The results of numerical simulation of the flow around the wing section with the SC1095 airfoil (based on the developed computational model) are presented below.

Figure 14 shows the velocity field distributions, Figure 15 shows the vorticity fields in the vertical plane of the WT symmetry in the presence of a wing section with the SC1095 airfoil set to the pitch angle $\alpha=10^{\circ}$ at Mach $\mathrm{M}=0.4$. The results illustrate the essence of the air flow in various areas of the WT and the rig, as well as in the area of the wing section. Analysis of the flow features makes it possible to analyze the effect of the WT and the experimental stand on the wing section flow. Modeling of various experimental rig configurations using the developed WT model allows determination of the most rational configuration for each specific case, in terms of reducing the impact of the experimental equipment on the results of the experiment. 

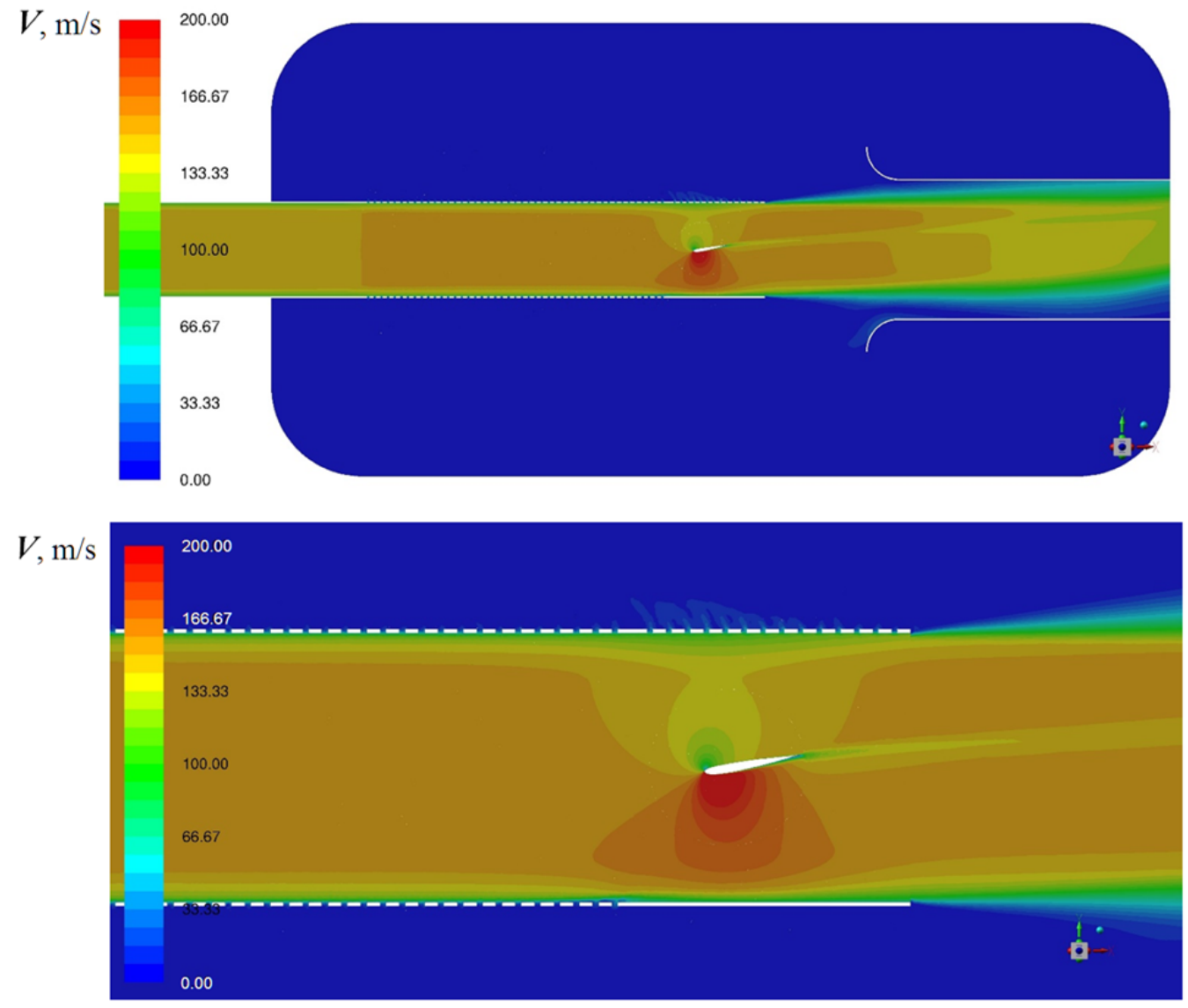

Figure 14. Distribution of the velocity field in the Eiffel chamber, in the working section and in the area of the wing section model at $\alpha=10^{\circ}$ and $M=0.4$

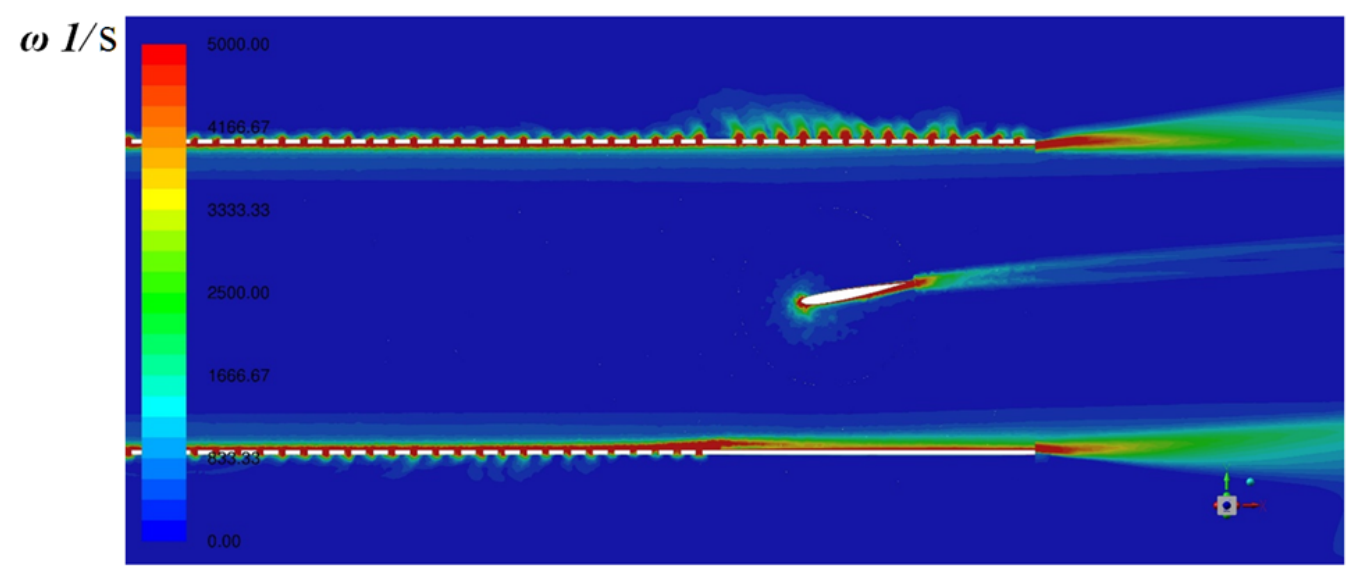

Figure 15. Distribution of the vorticity field in the Eiffel chamber, in the working section and in the area of the wing section model at $\alpha=10^{\circ}$ and $M=0.4$

Figure 16 and Figure 17 show the results of calculations of the steady aerodynamic characteristics of the wing section, obtained by using the developed WT model in a 3D setting, as well as the results of 2D calculations of the airfoil in free flow. The dependences of the lift and the drag force coefficient on the pitch angle are also presented. Calculations with the use of the WT model were performed for the Mach number $\mathrm{M}=0.4$ in the configuration of the experimental rig with a perforated top cover. The difference in the calculation results is due to the influence of WT and the rig. Using the developed WT model with different rig configurations, including different perforations, may help to determine the rig configuration and provide minimal impact on the model aerodynamic characteristics. The presented results can be an additional source for determination of corrections for recalculating the characteristics obtained in WT tests. 


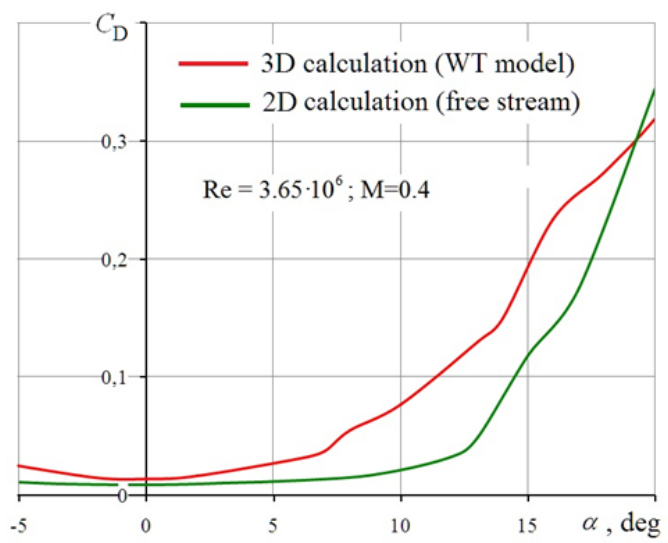

Figure 16. Dependence of the drag coefficient of the SC1095 airfoil on the pitch angle

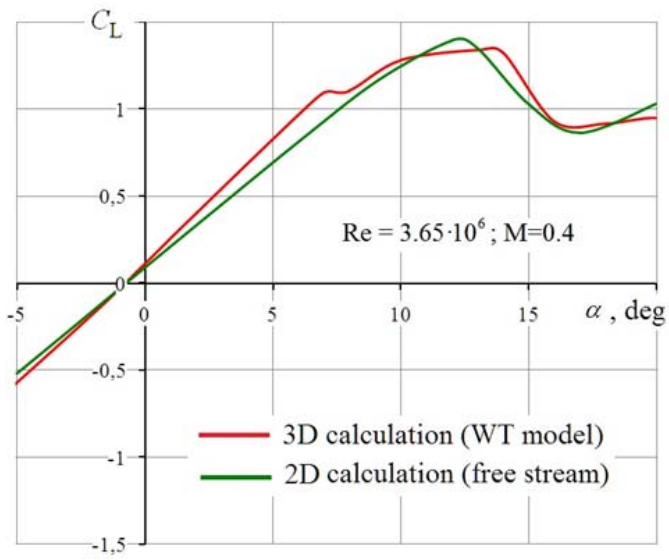

Figure 17. Dependence of the lift coefficient of the SC1095 airfoil on the pitch angle

Figure 18 and Figure 19 show the results of calculating the unsteady aerodynamic characteristics of the wing section using the developed WT and experimental rig models. Parameters of wing section fluctuations (1): vibration amplitude $A_{V}=5^{\circ}$, average pitch angle $\alpha_{\mathrm{av}}=10^{\circ}$, vibration frequency $f=5[\mathrm{~Hz}]$, Mach number $M=0.4$. The calculated values of the lift coefficients $C_{\mathrm{L}}$ and drag coefficients $C_{\mathrm{D}}$ for the Mach number $\mathrm{M}=0.4$ depending on the pitch angle are presented.

The graphs in Figure 18 and Figure 19 show the effect of dynamic flow around the wing section which is expressed through the hysteresis cycles.

Figure 18 shows that for the same pitch angles, when $\dot{\alpha}>0$ (the pitch angle increases), the stall is delayed, and when $\dot{\alpha}<0$ (the pitch angle decreases), the lift force decreases due to the flow stall on the profile. The greatest hysteresis is observed in the area of critical (stall) pitch angles.

Figure 19 shows that an increase in the pitch angle of the wing section $\dot{\alpha}>0$ leads to a sharp increase in the coefficient of drag as it approaches the critical pitch angle. In case of stall $\left(\alpha \approx 11^{\circ}\right)$, there is a sharp decrease in drag, followed by an increase as the pitch angle increases. During the return movement, when the pitch angle of the wing section decreases $\dot{\alpha}<0$, a monotonous decrease in the drag coefficient is observed.
These results indicate the need to single out changes in the aerodynamic characteristics of the profile in the case of dynamic flow conditions.

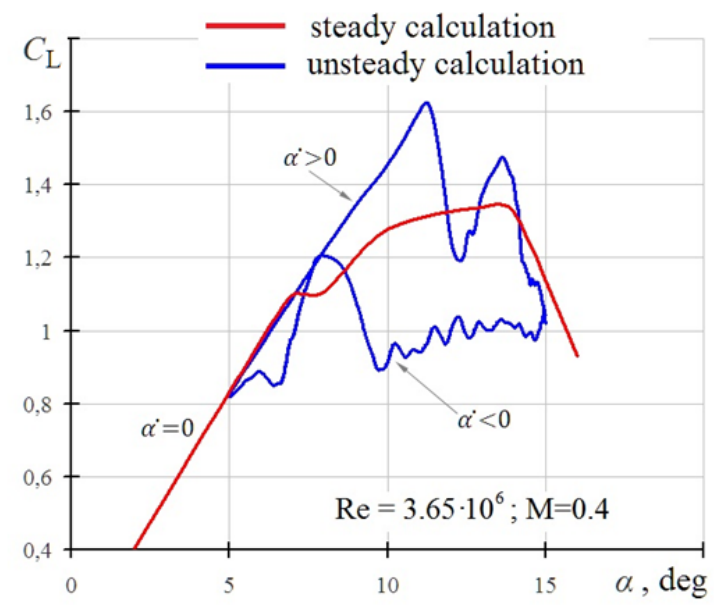

Figure 18. The dependence of the wing section lift coefficient on the pitch angle

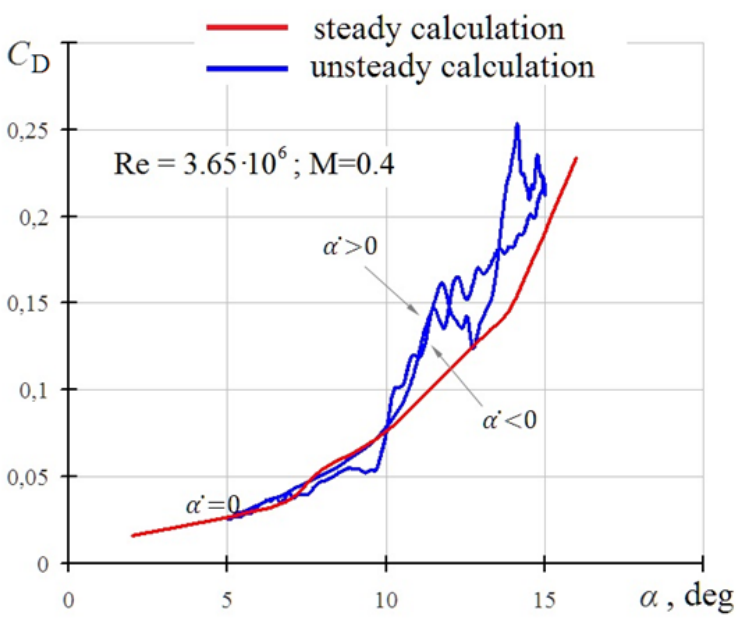

Figure 19. The dependence of the wing section drag coefficient on the pitch angle

\section{B) Conclusions}

The math model of the TsAGI WT with various options of the experimental rig for the study of unsteady aerodynamic characteristics of helicopter airfoils has been developed.

The model, based on the CFD URANS method, provides determination of the aerodynamic characteristics of the wing section in steady and unsteady settings. The model allows taking into account the influence of the WT and the experimental rig configurations, as well as conducting a complete analysis of the physical flow pattern of the studied wing section.

Validation of the calculation results of the steady aerodynamic characteristics of the SC1095 airfoil in 2D flow showed a satisfactory match of the calculated characteristics with experimental data in a wide range of pitch angles $-5^{\circ} \leq \alpha \leq 20^{\circ}$ at Mach numbers $\mathrm{M}=0.4$. 
The developed model makes it possible to determine rational parameters of the experimental rig configuration (including walls perforation parameters) based on studying the flow characteristics inside the working section of the WT. It leads to reducing WT influence on the conditions of the flow around the wing section.

Comparison of airfoil aerodynamic characteristics calculated on the basis of developed WT model and results of the calculations in the 2D-formulation in the free stream are different, due to the influence of WT and experimental rig. A comparative analysis of the results obtained can be used to determine the corrections for converting the results of experiments in WT to the full-scale characteristics of the airfoil.

Based on the developed model, the calculation of the unsteady flow around the oscillating wing section showed a significant difference in the steady and unsteady aerodynamic characteristics of the airfoil, which is expressed in the appearance of hysteresis cycles. It is necessary to take into account the unsteady flow of the airfoil of the rotor blade.

\section{References}

[1]. Johnson, W. (1986). Recent developments in rotarywing aerodynamic theory. AIAA journal, 24(8), 12191244.

[2]. Johnson, W. (2012). Helicopter theory. Courier Corporation.

[3]. Ahaus, L. A. (2010). An airloads theory for morphing airfoils in dynamic stall with experimental correlation (Doctoral dissertation, Washington University in St. Louis).

[4]. Golovkin VA, Kalyavkin VM (1974) Unsteady aerodynamic loads on the surface of the blade of a model of a heavily loaded main Rotor. Scientific notes of Central Aerohydrodynamic Institute, 3(5), 119-122.

[5]. Kirillov, O.E., Mirgazov, R.M., Golubev, N.V., \& Petruhin, D. A. (2019). Experimental studies of nonstationary aerodynamic characteristics of a helicopter airfoil vibrating in pitch angle. Trudy MAI, 105, 1-21.

[6]. Koning W.J.F., (2015). Wind Tunnel Interference Effects on Tilt Rotor Testing Using Computational Fluid Dynamics, Master's thesis at TU Delft, The Netherlands.

[7]. Decours, J.; Beaumier, P.; Khier, W.; Kneisch, T.; Valentini, M.; Vigevano, L.,(2014). Experimental validation of tilt-rotor aerodynamic predictions. In Proceedings of the 40th European Rotorcraft Forum, Southampton, UK, 2-5.
[8]. Olander, M. (2011). CFD Simulation of the Volvo Cars Slotted Walls Wind Tunnel. Göteborg: Master Thesis performed at Volvo Cars, Chalmers University of Technology.

[9]. Ljungskog, E., Sebben, S., \& Broniewicz, A. (2020). Inclusion of the physical wind tunnel in vehicle CFD simulations for improved prediction quality. Journal of Wind Engineering and Industrial Aerodynamics, 197, 104055.

[10]. Kalvig, S., Hjertager, B., \& Manger, E. (2014). Comparing different CFD wind turbine modelling approaches with wind tunnel measurements. In Journal of Physics. Conference Series (Online) (Vol. 555, No. 1).

[11]. Réthoré, P. E. M., Zahle, F., Sørensen, N. N., \& Bechmann, A. (2011). CFD Simulations of the Mexico Wind Tunnel and Wind Turbine. In EWEA Annual Event 2011. European Wind Energy Association (EWEA).

[12]. Qin, N. (2003). CFD for Better Understanding of Wind Tunnel Tests. In Integrating CFD and Experiments. Sheffield.

[13]. Vladimirova, N. A. (2015). Numerical simulation of the effect of permeable boundaries on transonic flow around a straight wing model in adaptive perforated wind tunnel. Proceedings of the XXVI scientific and technical conference on aerodynamics, Russia, Moscow region, Zhukovsky, 74-75.

[14]. Calautit, J. K., Chaudhry, H. N., Hughes, B. R., \& Sim, L. F. (2014). A validated design methodology for a closed-loop subsonic wind tunnel. Journal of Wind Engineering and Industrial Aerodynamics, 125, 180-194.

[15]. Sahin, S. E., Russell, C. R., Solis, E., \& Rajagopalan, G. R. (2016). Analysis of Large Civil Tilt Rotor Wind Tunnel Blockage and Validation Using RotCFD. AHS International Technical Meeting on Aeromechanics Design for Vertical Lift, San Francisco, California, January 20-22, 2016.

[16]. Ferziger, J. H., Perić, M., \& Street, R. L. (2002). Computational methods for fluid dynamics (Vol. 3, pp. 196-200). Berlin: Springer.

[17]. Schlichting, H (1979). Boundary Layer Theory. Seventh Edition. New York: McGraw-Hill.

[18]. Menter, F. R. (1994). Two-equation eddy-viscosity turbulence models for engineering applications. AIAA journal, 32(8), 1598-1605.

[19]. Bousman, W. G. (2003). Aerodynamic characteristics of SC1095 and SC1094 R8 airfoils. National Aeronautics and Space Administration Moffett Field Ca Ames Research Center.

[20]. Flemming, R. J. (1984). An experimental evaluation of advanced rotorcraft airfoils in the NASA Ames Eleven-Foot Transonic Wind Tunnel. NASA Contractor Report 166587. 